\title{
Barriers to Alzheimer Disease Drug Discovery and Development in Academia
}

\author{
\#*Linda J. Van Eldik, \#*Tanuja Koppal, and *†D. Martin Watterson \\ \#Departments of Cell and Molecular Biology, †Molecular Pharmacology and Biological Chemistry, and *Northwestern Drug \\ Discovery Program, Northwestern University Medical School, Chicago, Illinois, U.S.A.
}

\begin{abstract}
Summary: The drug discovery and the drug development processes represent a continuum of recursive activities that range from initial drug target identification to final Food and Drug Administration approval and marketing of a new therapeutic. Drug discovery, as its name implies, is more exploratory and less focused in many cases, whereas drug development has a clinically defined endpoint and a specific disease goal. Academia has historically made major contributions to this process at the early discovery phases. However, current trends in the organization of the pharmaceutical industry suggest an expanded role for academia in the near future. Megamergers among major pharmaceutical corporations indicate their movement toward a focus on end-stage clinical trials, manufacturing, and marketing. There has been a parallel increase in outsourcing of intermediate steps to specialty small pharmaceutical, biotechnology, and contract service companies. The new paradigm suggests that academia will play an increasingly important role at the proof-of-principle stage of basic and clinical drug discovery research, in training the future skilled work force, and in close partnerships with small pharmaceutical and biotechnology companies. However, academic drug discovery research faces a set of barriers to progress, the relative importance of which varies with the home institution and the details of the research area. These barriers fall into four general categories: (1) the historical administrative structure and environment of academia; (2) the structure and emphasis of peer review panels that control research funding by government and private agencies; (3) the organization and operation of the academic infrastructure; and (4) the structure and availability of specialized resources and information management. Selected examples of barriers to drug discovery and drug development research and training in academia are presented, as are some specific recommendations designed to minimize or circumvent these barriers. In some cases, precedents established by other disease-focused areas may be relevant to Alzheimer disease and related disorders, but the overall impact of any changes requires adaptation at the top of the administrative structures in academia and funding agencies to support and encourage cooperative efforts among faculty investigators. Key Words: Drug discovery and development-ChemistryNeurodegenerative disorders-Pharmacology.
\end{abstract}

Research in the L.J.V.E. and D.M.W. labs is supported, in part, by NIH grants AG13939 and AG15501, and by grants from the Alzheimer's Association and the Institute for the Study of Aging. T.K. was supported by NIH training grant AG00260. The resources cited in this article are not exhaustive, are intended as examples only, and do not represent an endorsement of any particular vendor.

Address correspondence and reprint requests to Dr. Linda J. Van Eldik, Northwestern University Medical School, Dept. of Cell and Molecular Biology, Ward 4-202, 303 E. Chicago Avenue, Chicago, IL 60611-3008, U.S.A.; e-mail: vaneldik@northwestern.edu.
Remarkable progress has been made during the past decade on understanding the multiple factors that contribute to different forms of Alzheimer disease (AD) and related neurodegenerative diseases. Recent insights into the pathobiologic mechanisms that underlie the onset and progression of the clinical course of the disease, although incomplete, are revealing specific molecules and pathways that are potential targets for new therapeutic ap- 
proaches (Cummings and Jeste, 1999). The state of the art in $\mathrm{AD}$ research is, therefore, squarely in the early phase of the drug discovery and development continuum.

The drug discovery and development processes represent two distinct but interdigitated aspects of research and engineering but are actually part of a continuum of recursive activities that range from basic science investigations and initial drug target identification to final Food and Drug Administration (FDA) approval, drug production under regulated conditions, and marketing of a new therapeutic agent (Fig. 1). Drug discovery, as its name connotes, is more exploratory and usually less focused than drug development, whereas drug development has a clinically defined endpoint and a specific disease goal. For example, the identification of a molecular target that is potentially amenable to AD drug development is a major effort and long-term goal of early drug discovery activities. These require the development of appropriate reagents and assays for the drug target and demonstration of its potential for modulation by chemical compounds. The details of early stage investigations are continually refined and potential targets are reevalu-

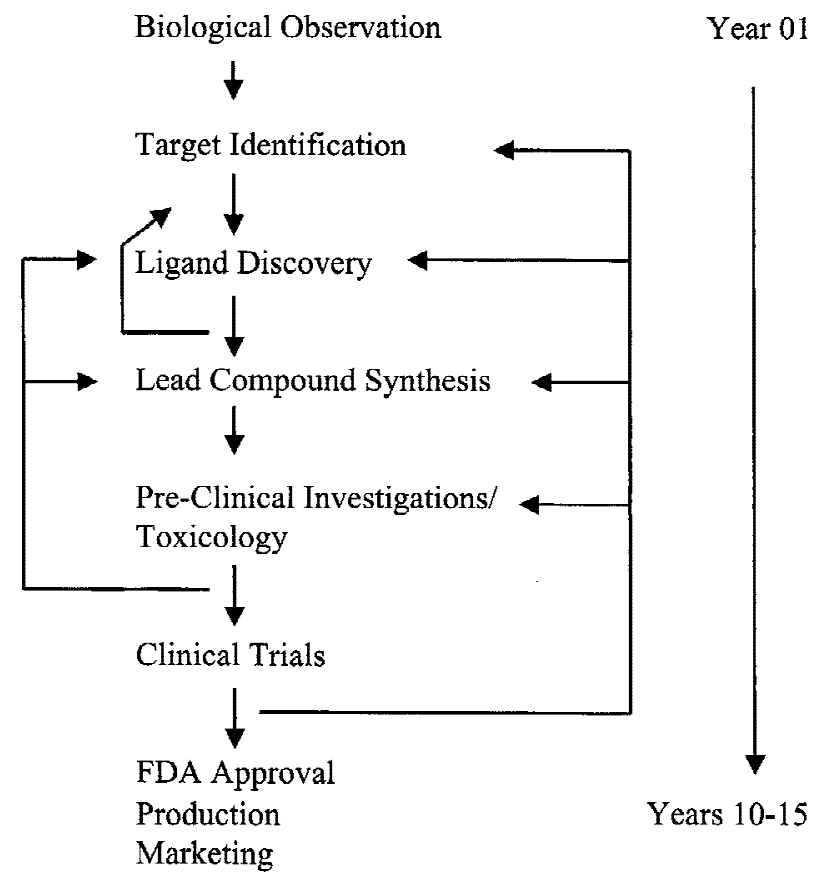

FIG. 1. The drug discovery-development continuum. The drug discovery and development processes represent a continuum of recursive activities that range from initial scientific observation and potential drug target identification to final Food and Drug Administration approval, drug production under regulated conditions, and marketing of a new therapeutic agent. The typical time for development of a new therapeutic agent is $10-15$ years. ated as ligands for modulation of biologic responses become available. In contrast, once a set of experimental drugs with a defined molecular target and potential for disease management are discovered, the development process has an almost institutionalized hierarchy and path that is followed to demonstrate safety and efficacy.

The decision by a major pharmaceutical company to fund a drug discovery and development project is an economic one based on a variety of criteria, including the realization that only a minor percentage of compounds coming out of drug discovery efforts become therapeutic agents and the need for future sales volume and low-cost production. The large pharmaceutical company must place an emphasis on shareholder return, deal with investor expectations, and support a large sales force and marketing costs. This consideration results in a comparatively conservative bureaucracy and risk-averse environment. The recent trend in megamergers among major pharmaceutical companies will probably make for an even larger bureaucracy and place more emphasis on production and marketing of compounds and therapeutic agents. This approach would focus a large, multinational pharmaceutical company's efforts on late stages of development and pose less risk to profit and shareholder returns. The void in the earlier phases of drug discovery and development that may be created by continuation of such trends among major pharmaceutical corporations can potentially be supplanted by the spin off of smaller and more specialized divisions, partnering with focused small drug development and biotechnology companies, and increased use of academic-based researchers or partnerships for early-stage discovery research.

The decision by a smaller pharmaceutical or biotechnology company to fund a project is determined by priorities related to its focused business plan and is significantly influenced by the expectations of its major investors. Small pharmaceutical or biotechnology companies tend to be more entrepreneurial and focused on applied research in specific franchise areas of technology, but they lack the infrastructure and experience of large pharmaceutical companies and must have a narrow focus on the number of projects and technology platforms that can be supported. There are recent changes, however, in which more research from large pharmaceutical companies is outsourced to companies specializing in particular technology platforms, and cooperative agreements on specific products or disease areas are drafted between complementary smaller companies or between smaller companies and major pharmaceutical corporations (Borchardt, 2000; Herklots, 2000). An example of a specialty company is MediChem, a drug discovery company that 
bridges the gap between new drug target identification and development of novel drug compounds by providing technology and services in proteomics, analytical and medicinal chemistry, biocatalysts, and process development. Another example is Prestwick, a company that specializes in enabling basic research results to proceed to end of phase II analysis by organizing and managing all aspects of development up to demonstration of efficacy in patients. Most recently, there is the trend of disease-focused foundations supporting small drug discovery or pharmaceutical companies to do early-stage drug development. Two examples are the Cystic Fibrosis Foundation's Therapeutics Development Program and the Institute for the Study of Aging Biotechnology Program for AD drug discovery and development.

The historical link between biotechnology or high technology companies and groups of major research universities provided an environment that allowed the development of geographical "hot spots" of entrepreneurial activity and translational activity in the application of basic research to products and the marketplace. The lessons learned from the actions and inactions of universities in this earlier phase of academia's evolution in the United States can provide a learning platform for potential evolution of academia's involvement in drug discovery and development. However, there are several differences in the paradigm for drug development, including the existence in academia of portions of the required infrastructure for drug discovery and development (especially in major academic medical centers or consortia among institutions), the history of cooperative faculty efforts, and the support of shared resources in the leading research institutions. An example of the emerging trend in academia is the cooperative support of a small group of scientists in the Northwestern Drug Discovery Program and investigators in the Institut Gilbert Laustriat at the Université Louis Pasteur in Strasbourg, France. This collaboratory, or infrastructures not bound by one set of walls, has been able to establish and screen pharmacologically "smart" chemical libraries containing hundreds to thousands of compounds each (composed of chemically diverse compounds used in previous drug development work), develop high throughput screening assays with mechanistic links to disease models and AD pathology, and initiate development of new lead compounds. These two academic groups have diverse links to small and major pharmaceutical companies for further development and potential commercialization. However, as with major pharmaceutical companies and smaller biotechnology companies, academia has a set of barriers to its increased participation in drug discovery and drug development research.

\section{HISTORICAL ADMINISTRATIVE STRUCTURE AND ENVIRONMENT OF ACADEMIA}

Academia is focused on education and exploratory research and has evolved an elaborate bureaucracy that is, like the large pharmaceutical corporations, conservative and risk averse. The culture is one that encourages the independent investigator and does not always facilitate or reward cooperative or group efforts in the life sciences. The recently completed genome sequence projects had a large academia component, but the majority of work was done at a limited few sites among collaborative groups dispersed over several institutions. The required infrastructure and funding were provided by extramural funding sources. The rewards for academic promotion and tenure were limited. In this regard, life science research in academia has not yet undergone the evolution seen in previous decades for the physical sciences and engineering, where collaborative group efforts and delayed, multiauthored publications are the norm for faculty promotion committees to review. The genome sequence projects were a watershed event for life sciences research in academia. The linkages and cooperation among geographically disperse groups of investigators documented the ability of such faculty-operated consortia to succeed.

Drug discovery and development research in academia is generally determined by the availability of funding from government agencies [e.g., the National Institutes of Health (NIH)] and disease-oriented foundations. Funding for late-stage drug discovery and early-stage drug development research in academia was rare or not available until recent changes and precedents. These included special requests for applications by the NIH or funding by foundations. However, the funding is still limited in amount and availability, and most research universities have been slow to respond to this changing paradigm. Historically, contributions from academia to the drug discovery and development continuum have been at the front end (e.g., the identification of a potential molecular target, development of appropriate reagents and assays for the target, and demonstration of its potential modulation in the appropriate biologic system), and funded by individual investigator-initiated grants. However, there is an evolving use of common technology platforms and experimental approaches in contemporary discovery and development phases. In addition, there is an increasing consideration of long-term goals in early phase research as a result of extensive use of informatics and enhanced academic training programs that target awareness about the drug discovery and development process. These recent changes increase the potential for academic scientists to contribute to the shortening 

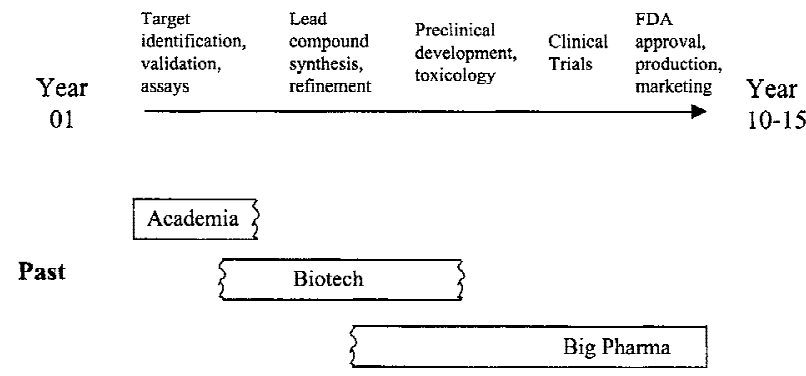

\begin{tabular}{|c|c|c|c|}
\hline Current & Academia & Biotech/Small Pharma & \\
\hline Future & & \{ & Big Pharma \\
\hline
\end{tabular}

FIG. 2. Trends in drug discovery and drug development. Historically, academia has contributed to the drug discovery-development continuum in the early discovery phases. The later stages, characterized by the drug development process, have been primarily the domain of the major pharmaceutical companies. However, current trends in the organization of the pharmaceutical industry suggest an expanded role for academia in the near future. Megamergers among major pharmaceutical corporations indicate their movement toward a focus on end-stage clinical trials, manufacturing, and marketing. There has been a parallel increase in outsourcing of intermediate steps to specialty small pharmaceutical, biotechnology, and contract service companies. This shifting paradigm suggests that academia will play an increasingly important role at the proof-of-principle stage of basic and clinical drug discovery research, in training the future skilled work force, and in close partnerships with small pharmaceutical and biotechnology companies.

of the time line for the early phase of the overall process and for academic-based cooperative efforts with industry in areas of orphan drug development, drug refinement, or proof-of-principle stages in development (Ryan, 2000; Ohlstein et al., 2000).

The trend of shifting relationships (Fig. 2) among major pharmaceutical corporations, biotechnology and drug discovery companies, and academia requires that the field draw increasingly on the entrepreneurial behavior of faculty and facilitate group interactions across multiple disciplines and institutions. The modified paradigm required of academia is important to the enhancement of a research system that will encourage new classes of therapeutic agents and novel treatments and the preparation of an appropriately trained scientific work force. The previous use of academic-based drug discovery and development research by industry has resulted in an increasing level of informal interactions between academia and industry during the past 20 years, with the importance of cooperative relationships between academia and industry becoming increasingly appreciated in the past 5 years. Academic training and educational programs are making concerted attempts to familiarize the next generation of scientists with the operations and goals of industry-based drug discovery and development and with the more theoretical, or proof-of-principle, goals of academia research.

Drug discovery and development programs in academia that train and foster collaborative research are being established. Some programs emphasize preclinical drug discovery and chemical biology and are research and training driven across multiple diseases (Table 1). Other programs focus largely on later-stage drug development and experimental therapeutic agents and are exemplified by clinical research and trials centers, diseasefocused consortia such as cancer centers or AD centers, and incubator resources (Table 1). These centers and programs in academia provide an environment and infrastructure that can encourage innovative and interdisciplinary research and foster more rapid progress in drug discovery efforts, provide a rich and stimulating educational environment, and facilitate communication and interactions between academia and industry. Directly relevant to the changing paradigm in the industry (Fig. 2), the establishment of early-stage discovery research and later-stage clinical trials and human biology research in medical center environments offers the opportunity for coverage of multiple points in the discovery and development continuum (Fig. 1) in collaboration with industry, or through major funding by the NIH or foundations if there is not sufficient profit potential for industry.

An important component for drug discovery and development research activities in academia is the university technology transfer office. These offices are often understaffed or underfunded within the university or have administrative charges that put them into potential

TABLE 1. Examples of drug discovery/drug development programs in academia

\begin{tabular}{|c|c|}
\hline $\begin{array}{l}\text { Drug Discovery and Chemical } \\
\text { Biology Research and Training } \\
\text { Programs (selected examples) }\end{array}$ & $\begin{array}{c}\text { Drug Development, } \\
\text { Experimental Therapeutics and } \\
\text { Incubator Resources (selected } \\
\text { examples) }\end{array}$ \\
\hline $\begin{array}{l}\text { Northwestern University Drug } \\
\text { Discovery Program } \\
\text { SUNY-Buffalo Center for Drug } \\
\text { Discovery and Experimental } \\
\text { Therapeutics } \\
\text { University of Florida Center for } \\
\text { Drug Discovery } \\
\text { University of South Florida } \\
\text { Cancer Drug Discovery } \\
\text { Program } \\
\text { University of Virginia Institute } \\
\text { for Structural Biology and } \\
\text { Drug Discovery }\end{array}$ & $\begin{array}{l}\text { Emory University Molecular } \\
\text { Therapeutics and Toxicology } \\
\text { Program } \\
\text { Johns Hopkins Cancer Drug } \\
\text { Development Training } \\
\text { Program } \\
\text { Northwestern University Clinical } \\
\text { Trials Research and Training } \\
\text { Center } \\
\text { University of Kansas Higuchi } \\
\text { Biosciences Center of } \\
\text { Excellence } \\
\text { University of Pittsburgh Drug } \\
\text { Development Program }\end{array}$ \\
\hline
\end{tabular}



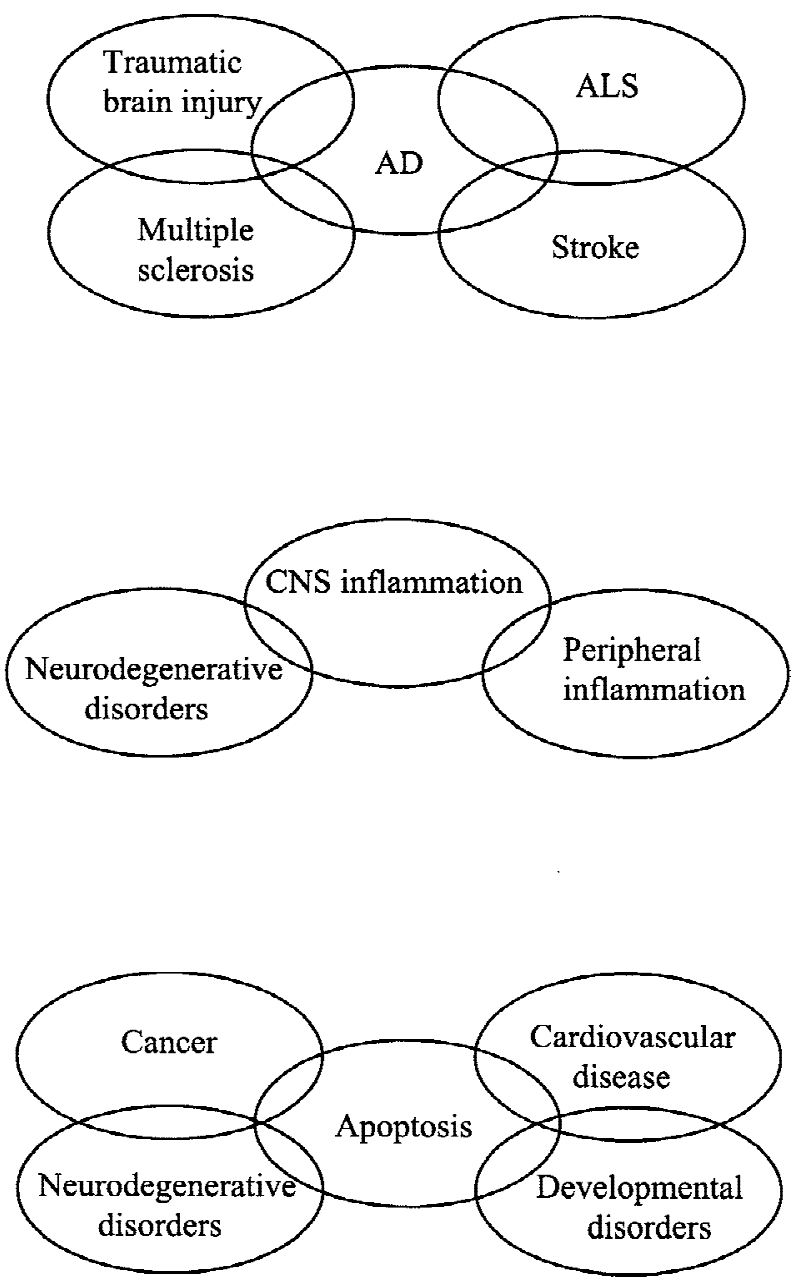

FIG. 3. Overlap of knowledge and approaches across multiple disease areas. Much of the early-stage drug discovery knowledge gained and approaches developed in one disease-oriented area can be relevant to other disease areas. For example, discovery of potential molecular mechanisms of neurodegeneration in $\mathrm{AD}$ is likely to be relevant to multiple diseases in which neurodegeneration is a component, such as amyotrophic lateral sclerosis, traumatic brain injury, stroke, and multiple sclerosis. Similarly, insight into mechanisms of neuroinflammation may be important for neurodegeneration and for the broader class of peripheral inflammatory diseases, whereas elucidating pathways of programmed cell death (apoptosis) may have relevance to neurodegenerative disorders and to development-related disorders, cardiovascular diseases, and cancer.

conflict with the rapid advance of science by the faculty investigators. These diverse problems that vary with each institution have been addressed by many universities and professional associations involved with this area of academia operations. Intellectual property offices are becoming, in some cases, catalysts for progression of drug discovery research results into early-stage drug development research or its transfer to industry. Therefore, the more sophisticated and experienced intellectual property offices in major research universities enhance the poten- tial financial and intellectual impact of drug discovery research for the university and remove a major barrier to drug discovery and development research in academia.

Relevant to intellectual property and insight into disease mechanisms and therapeutic development, much of the knowledge gained and approaches developed in one disease-oriented area can be transferred successfully to another field of research, such as AD (Fig. 3). This influence can readily be seen in industry and academia at late-stage drug discovery and early-drug development phases. For example, the current excitement about proteases in apoptosis (caspases) and amyloid processing (secretases) will benefit greatly from the recent past success in targeting proteases in AIDS therapeutics development (Korant and Rizzo, 1997; Lin, 1997; Ren and Lien, 1998; Sommadossi, 1999). Much has been learned about how to inhibit selectively these proteases and which pharmacologic features are important for protease inhibitors in terms of adsorption-distribution-metabolism-excretion (ADME) profiles and toxicology. This example and other potential transference of knowledge and approaches can potentially be applied to AD drug discovery and development targeting proteases. In addition, early-stage drug discovery research focused on AD has the potential, in turn, for contribution to the study of other diseases. For example, discovery of potential molecular mechanisms of neurodegeneration in $\mathrm{AD}$ is likely to be relevant to multiple diseases in which neurodegeneration is a component, such as amyotrophic lateral sclerosis (ALS), traumatic brain injury, stroke, and multiple sclerosis. Similarly, insight into mechanisms of neuroinflammation may be important for neurodegeneration and for the broader class of peripheral inflammatory diseases, whereas elucidating pathways of programmed cell death (apoptosis) may have relevance to neurodegenerative disorders and to development-related disorders, cardiovascular diseases, and cancer.

Foundations, the NIH, and industry are the financial sources of the continuing role of academia in drug discovery and development research in selected diseaseoriented areas, such as AD. Disease-targeted NIH funding of clinical research areas and the recent organization of industry-sponsored drug trial centers in academic medical centers are increasing the participation of academia in the later stages of drug development, complementing the historical participation of academia in earlystage drug discovery research. As examples, the networking of $\mathrm{AD}$ centers and their various consortia and collaborative studies and clinical therapeutics cooperatives among comprehensive cancer centers (and other disease-targeted initiatives) have gone a long way toward 
alleviating barriers at the clinical research end of the spectrum.

What has been insufficient in the recent trends in extramural funding of academic drug discovery research includes (1) support of investigator-initiated research on cross-cutting technology platforms and scientific endeavors that have potential impact on the drug discovery and development process across multiple disease areas in the longer term (but may not be specific disease focused in the shorter term); (2) support of cooperative infrastructures needed for late-stage drug discovery and earlystage drug development in academia; and (3) seed funding of hypothesis-driven, high-risk ventures by collaborations among academic-based investigators, especially ligand discovery research. In many cases, the barriers to drug discovery and development research in academia at this stage are a reflection of the barriers to research at the chemistry-biology interface in academia. This finding is especially acute at the preclinical research level.

Because of academia's dependence on external funding of research as a major income source, NIH funding initiatives have a major impact on the operations, infrastructure, and decision-making processes in academia. Therefore, some barriers to drug discovery and drug development research in academia could be potentially addressed across many institutions by reviewing funding mechanisms. Because of their role as driving forces on academia and their potential as existing mechanisms for immediate action to stimulate and facilitate AD drug discovery and development research in academia, the funding priorities and peer review processes of NIH represent the most immediate barriers to academic drug discovery to address. However, the context of academia structure and administration is a more fundamental barrier that varies in degree between institutions.

\section{Barriers}

The historical administrative structure and environment of academia limit performance of drug discovery and development projects because of the multidisciplinary nature of such research and the associated engineering-related activities; academia is still mostly organized and functions along classic disciplinary lines, especially in the career advancement of faculty.

Rapid advances in basic science and technology increasingly make the important scientific questions ones that are concerned with problems rather than disciplines, and addressing these questions demands interdisciplinary approaches. Universities, with their traditional vertical departmental structure and hierarchal university administrative structures, generally have difficulty implementing and coordinating interdepartmental endeavors and rapidly responding to progress in a problem-solving endeavor. There is a lack, especially in the basic sciences, of alternative career opportunities in academia for scientists and engineers outside the classic lecture-based and tenure-track-associated career paths. Highly skilled and motivated persons interested in these alternative career paths are crucial to a major drug discovery and drug development effort in academia. In addition, universities do not necessarily value applications-oriented or collaborative endeavors that cross the usual disciplinary boundaries. Therefore, the typical academic structure in the United States can be a major barrier to drug discovery and development research in academia.

An evaluation parameter that should be noted in advance by funding agencies interested in supporting drug discovery and development research in academia is the administrative mechanisms for gaining acceptance of faculty-initiated efforts or allowing more direct faculty participation in the university enterprise when research depends on cross-disciplinary efforts. For example, there needs to be encouragement and support for establishment of basic science-based drug discovery programs that are interdepartmental and interdisciplinary and that interdigitate with multiple disease-targeted clinical centers. A complementary need is for academic institutions to support drug development-oriented research in diseasefocused clinical centers, with close collaboration between basic and clinical scientists. Related to these research-intensive efforts at the biology-chemistry interface, support for applications-oriented synthetic chemistry faculty in a medical center environment would be a tremendous catalyst for enhancement of diseaseoriented drug discovery research in academia. Development of multidisciplinary research at the chemistrybiology interface and integration of engineering and mathematics into the traditional life sciences will also require new initiatives in graduate education that emphasize these information-driven coalitions and systems approaches. These changing definitions of graduate training and support for such integrative training initiatives are beginning to be catalyzed by NIH and other organizations, such as the Howard Hughes Medical Institute, which is helping to circumvent the administrative structure barrier to drug discovery in academia.

A closely related academic barrier, especially in private university environments, is that there may be only limited institutional enthusiasm for endeavors that do not result in full recovery of administrative charges (e.g., overhead or indirect costs) or that detract from clinical service income. These are economic obstacles that are difficult to overcome. However, the emphasis at extramural funding agencies, such as the NIH, on investiga- 
tor-initiated grants with full administrative cost recovery has a proven track record of success as a strategy to encourage fundamental research within a particular field and is an important mechanism for encouragement of drug discovery and early-stage drug development research in academia. Similarly, the de facto peer review pressure for program project and other multiinvestigator grants with full administrative cost recovery to be multidepartmental can also lessen barriers in this category. For example, a National Institute on Aging (NIA) program announcement in 1999 (PAS-99-034) invited program project grant applications specifically in the area of drug discovery for the management of AD. This approach is an excellent start to encourage drug discovery research in $\mathrm{AD}$. Increasing the funding of various other kinds of cooperative endeavors in the area of AD drug discovery should also be considered. Other diseasefocused institutes have implemented a variety of funding initiatives in the area of drug discovery and development. Examples of some of the relevant programs developed by the National Cancer Institute are listed in Table 2. These precedents may provide help to determine what mechanisms of funding have been more successful than others. Some of these kinds of programs may work better for $\mathrm{AD}$ drug discovery and development, which has different demands on therapeutic indices than cancer drugs.
It should be emphasized, however, that the highest priority should be to increase the level of support for individual investigator-initiated research grants and program project grants in the area of AD drug discovery and drug development. The academic realities are such that increased extramural funding of AD-related drug discovery and drug development is an immediate and ADfocused mechanism for alleviating some of the inherent academic institutional barriers.

\section{STRUCTURE AND EMPHASIS OF PEER REVIEW PANELS}

Review panels as currently constituted do not necessarily have a full appreciation for the multidisciplinary, cross-disease research emphasis that is at the heart of drug discovery research or for the inherent barriers to those who seek to cross disciplinary or institutional boundaries to discover potential new therapies, or insight into alternative approaches to therapy, in the absence of the focused infrastructure found in industry.

The use of established funding mechanisms as an approach to minimizing major and fundamental barriers to AD research in drug discovery and development in academia depends on the availability of appropriately constituted peer review groups (e.g., study sections) with members containing the necessary scientific, technical,

TABLE 2. Examples of NCI funding initiatives/resources in drug discovery and development

\begin{tabular}{|c|c|}
\hline National Cancer Institute Drug Discovery/Development Programs & Purpose \\
\hline Molecular Target Drug Discovery for Cancer RFA-CA-00-002 & $\begin{array}{l}\text { For early stage discovery work; i.e. to identify, validate and } \\
\text { develop assays for novel molecular targets for cancer }\end{array}$ \\
\hline Assessment Program RFA-CA-00-001 & $\begin{array}{l}\text { mechanism assessment in clinical trials and preclinical cancer } \\
\text { models }\end{array}$ \\
\hline National Cooperative Drug Discovery Groups (NCDDGs) & To encourage cooperative research between academic and \\
\hline RFA-CA-99-010 & $\begin{array}{l}\text { industry-based investigators for drug discovery and } \\
\text { development }\end{array}$ \\
\hline Drug Synthesis and Chemistry Branch & To support acquisition and screening of compounds and \\
\hline http://dtp.nci.nih.gov/docs/cancer/cancerscreen.html & combinatorial libraries \\
\hline Development Therapeutics Program (DTP) http://dtp.nci.nih.gov/ & For development of antineoplastic agents \\
\hline Rapid Access to Intervention Development (RAID) program & For rapid movement of novel molecules and concepts from \\
\hline http://dtp.nci.nih.gov/docs/raid/raid-index.html & $\begin{array}{l}\text { laboratory to clinic for proof-of-principle trials, supporting the } \\
\text { development of select compounds from academia }\end{array}$ \\
\hline $\begin{array}{l}\text { Rapid Access to NCI Discovery Resources (RAND) program } \\
\text { http://dtp.nci.nih.gov/docs/rand.html }\end{array}$ & $\begin{array}{l}\text { To facilitate access of academic centers to NCI resources for the } \\
\text { discovery and early preclinical development of drugs and } \\
\text { biologics }\end{array}$ \\
\hline $\begin{array}{l}\text { Rapid Access to Prevention Intervention Development (RAPID) } \\
\text { program http://www3.cancer.gov/prevention/rapid/ }\end{array}$ & $\begin{array}{l}\text { To support preclinical development of chemopreventive agents } \\
\text { and clinical development through Phase I studies }\end{array}$ \\
\hline Biological Resources Branch http://web.ncifcrf.gov/research/brb/ & $\begin{array}{l}\text { A directory of research tools and services for drug discovery } \\
\text { researchers }\end{array}$ \\
\hline Mouse Cancer Models Repository & To facilitate the development and validation of mouse models of \\
\hline http://web.ncifcrf.gov/researchresources/mmhcc/default.asp & $\begin{array}{l}\text { human cancer and to accelerate their dissemination to the } \\
\text { research community }\end{array}$ \\
\hline Molecular Targets Laboratory Initiative & To develop a resource of biological assays and chemical probes \\
\hline http://www3.cancer.gov/initiatives/grp-target.html & for cancer studies \\
\hline Web/accessible data and tools http://dtp.nci.nih.gov/webdata.html & Search tools and downloadable bulk data \\
\hline
\end{tabular}


and institutional experiences. Peer review groups are often oriented along classic disciplinary lines, which can result in difficulties for drug discovery and development proposals, especially at the basic science level. For example, high-risk exploratory synthetic chemistry proposals focused on ligand or inhibitor discovery can be viewed, in some cases, as "fishing expeditions" or "premature" if reviewed by a biology group. Even if feasibility data are outstanding and extensive, there can be concerns about funding such endeavors before commercialization is assured. If the review panel is in molecular biophysics, it may have less enthusiasm for a proposal with an aim to determine the three-dimensional structure of an enzyme as the starting point for structure-assisted inhibitor design if there is no chance to discover a new protein fold or if the enzyme is not the first in its class to be studied, even if it is the accepted drug discovery target. Similarly, a chemistry review group may lack enthusiasm for or appreciate the significance of a standard set of applications-oriented organic syntheses that do not allow much potential for new synthetic schemes or products. Special emphasis panels, however, can also present problems, especially if they focus too short term on disease relevance. For example, chemical synthesis and target structure projects could be viewed as "premature" if there is no clinical evidence for these pathways or targets being potentially relevant. Similarly, there can be hesitation to proceed if there is no existing genetically engineered mouse that fits the disease paradigm. These different areas of emphasis impede the discovery of novel therapeutic agents and alternative approaches by requiring a strong link to retrospective public health data and clinical correlations or by focusing only on established targets for the given disease.

There is great potential for academic scientists to explore regulatory pathways and their potential modulation by ligands as a result of the tremendous database of information about potential drug discovery targets coming out of genomics and proteomics research, the availability of new computational tools for ligand design, and the development of highly efficient and automated synthetic chemistry methods and genetics-style, highthroughput activity screens. In academia, the term chemical biology has gained popularity for classification of much of the fundamental research in this area (Walsh, 1998; Wells, 1999). If longer-term disease application is a goal of the research, then chemical biology is definitive early-stage drug discovery research.

Overall, there is a tendency for discipline-based review groups to lack appreciation for the significance of "applications-oriented" projects and for disease-focused review groups to want extensive feasibility data relevant to a particular disease before the funds are awarded. Study sections have tried to address this problem for drug discovery and development by inclusion of members from industry, but this partial solution is inadequate because these investigators may not fully appreciate what is reasonable to ask for in terms of feasibility data from an academic-based researcher. It is also important to note that academic-based reviewers with limited knowledge of the drug discovery and development process can also fail to appreciate the significance of the proposed research, even if they are able to evaluate the technologies. Therefore, experience and familiarity with drug discovery and development in academia are key criteria for the selection of appropriate reviewers for applications that propose AD drug discovery and development research in academia, even if the reviewer's disease focus is outside that of $\mathrm{AD}$ or related disorders.

\section{ORGANIZATION AND OPERATION OF THE ACADEMIC INFRASTRUCTURE}

The infrastructure in academia is generally inadequate to support late-stage drug discovery research or ADfocused drug development efforts. Outsourcing of key functions is an emerging trend in academia; multiinstitutional consortia and resources available for AD drug discovery and development efforts in academia are limited; and the potential for extramural funding for such infrastructure is inadequate.

For effective late-stage drug discovery and development efforts in academia, it is necessary to expand beyond the expertise and resources of one investigator because no single principal investigator and laboratory have the required expertise across multiple disciplines. There is a need for centrally supported resources for investigators to have access to rapid, high-quality results required for progress in basic and preclinical drug discovery research. It is understandable that one academic institution would be hesitant to invest heavily in the physical and personnel resources required for a minimally adequate infrastructure for each disease area. As in industry, successful AD drug discovery research and potential AD drug development in academia require collaborations among biologists, chemists, structural biologists, and clinicians and the availability of cost-effective, reliable support resources. Successful pharmaceutical firms have integral and highly expert groups whose function is defined as serving the generation of profits from cost-effective production of a drug with a significant market. These elaborate and highly specialized areas of expertise are necessary for a group-focused effort in which individual reward is directly linked to group success. Therefore, a current limitation in terms of critical 
mass of investigators and resources that is a barrier to $\mathrm{AD}$ drug discovery and development research in academia is the lack of major infrastructures required at selected stages of the research by a variable number of investigators or investigator groups. However, there are potential ways to alleviate these barriers immediately with coordinated action and participation of extramural funding agencies.

At the clinical therapeutics end of academic-based drug development research, there is already an excellent start for evaluation of therapies related to $\mathrm{AD}$ through the AD Cooperative Study, an NIA-sponsored consortium of academic AD medical research centers with a mandate to develop and conduct clinical trials for promising agents designed to ameliorate behavioral symptoms, improve cognition, slow the rate of decline, or delay the appearance of AD. This setup is a ready-entry mechanism for collaboration between clinician investigators and industry, including hypothesis-driven studies of existing pharmaceutical agents for alternative uses. However, there is a major barrier at the preclinical level in terms of infrastructure for pilot animal toxicology and feasibility testing in animal models of disease and scaled-up synthesis of compounds for therapeutic or diagnostic use.

Academia has moved in recent years toward the use of institutional core service laboratories or centrally supported resources operated on a fee-for-service or modified "open shop" structure so that investigators, or investigator groups, would have access to standardized, reproducible, and cost-effective resources required for effective progress in drug discovery research. Costeffective outsourcing arrangements with particular vendors have been established by some institutions. Examples of such resources that would be relevant to drug discovery are cores for animal models of disease and testing (Finch, 1999), synthetic chemistry and analytical chemistry service laboratories, structure determination service facilities, and other technology and informatics support cores. These resources are sometimes operated at a diversity of academic institutions in an efficient manner and are continually evaluated and restructured depending on the need of the institution. However, these cores can easily become inefficient and nonresponsive structures or become dominated by one major user group if there is not a development and evaluation plan based on end-user input and oversight for such cores and their associated staff. In addition, there must be some level of institutional commitment to the underwriting of individual services and resources. The structure of these resources and the availability of faculty committed to drug discovery and drug development research in academia will, in large part, determine the institution's support and tolerance of the infrastructure needs of investigators who must use multidisciplinary approaches. Although some variability must be available based on the details of the research programs and goals, the core and cross-disease infrastructure issues can easily be facilitated by sets of standardized guidelines and support mechanisms. Another problem is that the management of the infrastructure in academia is such that the highly skilled scientists and engineers needed for well-run support cores do not have viable alternative career paths in academia, with a resultant high turnover of the best persons as they leave scientific research in academia for other careers.

What has been emerging in recent years in response to problems in shared resources and infrastructure issues within an institution is the outsourcing of services to specialty biologic and chemical services corporations. For example, commercial firms have emerged in the past few years for animal services such as animal care and production of animal models of disease (see, for example, Charles River Laboratories at http://www.criver/com). Similarly, commercial synthetic chemistry specialty companies are now commonplace, and fee-for-service structure determination of drug discovery targets is a reality (e.g., MediChem Research, Inc.). These infrastructure-related services are cost and time effective (Borchardt, 2000; Herklots, 2000), especially if there is the need to determine structure only as the start of structure-assisted ligand design or to make one, well-designed knockout or transgenic mouse. However, outsourcing of routine analyses to service companies with specialized biologic and chemical expertise is not always recognized as a valid approach by extramural funding agencies. There is a need for funding agencies to recognize the reality of these infrastructure services and their advantages and to support them as part of research grant funding. In many cases, this support can be an efficient use of funds by the funding agencies and a rather straightforward way to remove a major barrier to AD drug discovery and development research in academia.

In the area of cancer, the National Cancer Institute has played a role in minimizing this infrastructure barrier by establishing several programs that provide researchers with access to resources for molecular target characterization and biomarker validation, acquisition of compounds and combinatorial libraries, mouse cancer models, biopharmaceutical production, and preclinical development of drugs and toxicology testing (Table 2). Although the cost-to-benefit ratio of these resources for the successful development of new anticancer therapeutic agents has been debated (Boyd and Paull, 1995; Report of the NCI Developmental Therapeutics Program Review Group, 1998), these programs provide a prece- 
dent for academic investigators being provided with resources and infrastructure that are not generally established in a university setting. Relevant to $\mathrm{AD}$, the NIA supports repositories of AD-related material, including an aging cell repository, non-human primates, and aged rodent colonies (http://www.nih.gov/nia/research/). This model of NIA support for AD-relevant resources should be continued and expanded. For example, for more effective $\mathrm{AD}$ drug discovery and development in academia, it would be extremely useful to have a centralized resource for maintenance and testing of relevant transgenic mouse models of aging and AD (Finch, 1999). An "animal models" core would provide a standardized resource for maintenance and distribution of wellcharacterized and uniform animal models to the AD research community and could provide preclinical testing of promising lead compounds in terms of behavioral, pathologic, and biomarker analyses. Encouragingly, the NIA has identified the continued development and enhancement of research resources, such as animal models, chemicals, imaging technologies, and informatics methodologies, as one of its goals in its Strategic Plan for the years 2001-2005.

Individual investigators and small groups of investigators have also addressed the infrastructure barrier through the development of informal cooperatives or collaborative relationships (e.g., collaboratories, or infrastructures not bound by one set of walls), including academic-based drug discovery and experimental therapeutics programs and interinstitutional linkages of research groups. This collaboration is a need-driven invention by the investigators. However, it is sometimes difficult to obtain recognition of these networks by either academic institutions or funding agencies. This barrier to diseasetargeted drug discovery and development research in academia can be effectively removed by recognition and support of the networks by the funding agencies. For example, if two research groups interested in discovery of ligands for modulation of biologic responses potentially linked to $\mathrm{AD}$ and other neurodegenerative disorders are sharing their chemical libraries and assays in a "virtual biology core" and a "virtual chemistry core," this collaborative effort should be recognized by the funding agency as appropriate for support. There should also be encouragement of specialized multiinstitutional shared resources by the funding agencies and institutions and consideration of academia-industry cooperatives (e.g., a set of universities with a biotech company) and academia-national laboratory cooperatives (e.g., universities and Department of Energy personnel at a national laboratory). Innovative and efficient solutions to infrastructure barriers, especially those established by the investi- gators, need to be recognized and financially underwritten by the funding agencies.

\section{STRUCTURE AND AVAILABILITY OF SPECIALIZED RESOURCES AND INFORMATION MANAGEMENT}

The coordinated and centralized information management of disease-relevant research results is inadequate.

The rapid advances in communications and information technologies and the globalization of science offer expanding opportunities for interinstitutional cooperation in research and education within academia and between academic institutions and other sectors. The impact of the National Library of Medicine information management facilities on international science through their access via the Internet and their increased use in the post-genome sequence era is an example of information management making a major contribution to international progress in biomedical research. The evolution of database management structures and user-friendly interfaces for sequence databases and macromolecular databases has been a great facilitator of drug discovery and drug development research for $\mathrm{AD}$ and other diseases. This development has an appropriate and valuable role. The public pressure on corporations to make their data available eventually on a shared basis in return for their access to the public information that formed the basis of much of the genome projects is an example of the impact of such resources on commercial activities. However, there is currently a lack of standardized requirements and methods of reporting on disease-relevant expression patterns in genomic studies (e.g., array data obtained for response to an AD-relevant drug) or proteomic investigations (e.g., changes in protein interactions in ADrelevant pathways). This lack of standardization results in missed information and in growing proprietary databases that vary in experimental design and control samples and promotes an environment in which many studies are repeated unnecessarily. A major barrier to $\mathrm{AD}$ drug discovery and development in academia is the lack of access and formalized mechanisms for distribution of this rapidly accumulating body of information that would facilitate drug discovery and development research. These are just examples, but there are diverse areas where facile information availability may stimulate the rate of discovery and promote collaborations and interactions among investigators from divergent and complementary backgrounds. This availability is key to the interdisciplinary interactions that are needed for drug discovery and drug development research.

A related barrier is the occasional lack of access to appropriate reagents needed for AD drug discovery re- 
search or the lack of reagents and services of uniform quality and proven reliability (e.g., difficulties in obtaining particular clones, antibodies, or animal models relevant to $\mathrm{AD}$ research and difficulties in obtaining synthetic $\beta$-amyloid peptides of high quality and consistency).

An excellent start to circumventing these barriers is the Alzheimer's Research Forum, a web site dedicated to enhancing information access and promoting scientific collaboration. This site provides several online tools and a compendium of AD-related resources available to researchers in the field. For example, there is an online AD clinical trials database; a listing of AD-related patents, publications, and conferences; and a directory of sources of antibodies, clones, tissue, and transgenic animals. Another useful web site is the Alzheimer's Disease Education and Referral Center, which provides $\mathrm{AD}$ information and referral services, news from NIA, and a list of ADrelated publications and clinical trials. These sites are two examples of an effective system for dissemination of useful AD-relevant scientific information to investigators. The next step required for more rapid progress in $\mathrm{AD}$ drug discovery and development in academia is a centralized and uniform system for facilitating interactions between discoverers and organizations who may be interested in developing such discoveries, or for providing resources to the investigator of early-stage development projects (e.g., support of a centralized AD research reagent and animal models repository, establishment of resources for preclinical development and testing of drugs and biologics, etc). NIH or other funding agencies could assist in overcoming these barriers by taking the lead in coordinating systems for more effective communication and distribution of $\mathrm{AD}$-relevant information and reagents and in facilitating interactions between academia and potential industrial partners.

\section{SUMMARY RECOMMENDATIONS}

- Establish basic science-based drug discovery programs in academia that support a variety of technology programs across multiple academic disciplines and that interdigitate with disease-targeted clinical centers

- Establish drug development-oriented research in disease-focused clinical centers, with close collaborations between basic and clinical scientists

- Establish peer review panels with appropriate expertise and experience in academic drug discovery and development research
- Use existing funding mechanisms to support AD drug discovery and development research in academia

- Acknowledge and support innovative investigatorinitiated solutions to academia infrastructure problems, including cooperative collaboratories, interinstitutional linkages of research groups, and outsourcing of specialty services

- Recognize the need for support of applicationsoriented synthetic chemistry efforts in medical center environments

- Appreciate and support, through recognition by academia, peer review groups, and funding agencies, the critical importance of alternative track academic researchers in $\mathrm{AD}$ drug discovery and development research

- Coordinate at the national level key information and specialty reagents and services relevant to $\mathrm{AD}$ drug discovery and development research

Acknowledgments. The authors thank their Drug Discovery Program colleagues and consultants for stimulating discussions about drug discovery and drug development in academia.

\section{REFERENCES}

Borchardt JK. Playing the economics game with outsourcing. Modern Drug Discovery 2000; March: 28-34.

Boyd MR, Paull KD. Some practical considerations and applications of the National Cancer Institute in vitro anticancer drug discovery screen. Drug Dev Res 1995;34:91-109.

Cummings JL, Jeste DV. Alzheimer's disease and its management in the year 2010. Psych Services 1999;50:1773-7.

Finch CE. Infrastructure for research on aging rodents: need for regional facilities to support transgenic studies on aging. Neurobiol Aging 1999;20:213-5.

Herklots H. Oursourcing the search for leads. Modern Drug Discovery 2000; March:46-50.

Korant BD, Rizzo CJ. The HIV protease and therapies for AIDS. Adv. Exp. Med. Biol. 1997;421:279-84.

Lin JH. Human immunodeficiency virus protease inhibitors. From drug design to clinical studies. Adv Drug Delivery Rev 1997;27:215-33.

Ohlstein EH, Ruffolo RR, Elliott JD. Drug discovery in the next millenium. Ann Rev Pharmacol Toxicol 2000;40:177-91.

Ren S, Lien EJ. Development of HIV protease inhibitors: a survey. Prog Drug Res 1998;51:1-31.

Report of the NCI Developmental Therapeutics Program Review Group, 1998. Available at http://deainfo.nci.nih.gov/advisory/bsa/ bsa_program/bscdevtherprgmin.htm. Accessed March 21, 2001.

Ryan JF. The pharmaceutical century. Ten decades of drug discovery, American Chemical Society, Washington, D.C. 2000.

Sommadossi JP. HIV protease inhibitors: pharmacologic and metabolic distinctions. AIDS 1999;13(suppl 1):S29-40.

Walsh C. Chemical biology: information, mesoscale science and the engineering ethos. Chem Biol 1998;5:R177-9.

Wells W. Chemical biology: the promise, and confusion, of adolescence. Chem Biol 1999;6:R209-11. 